\title{
Development of an Algorithm to Determine the Liquid Spreading Area of Airlaid Nonwoven Fabrics by Image Processing Method
}

\author{
H.İbrahim Çelik ${ }^{1}$, Elif Gültekin ${ }^{1}$, Serkan Nohut ${ }^{2,3}$ \\ ${ }^{1}$ Gaziantep University, Faculty of Engineering, Textile Engineering Department, Gaziantep, Turkey \\ ${ }^{2}$ Selçuk İplik San. ve Tic. A.Ş., Research and Development Center, Gaziantep, Turkey \\ ${ }^{3}$ Piri Reis University, Faculty of Engineering, Mechanical Engineering Department, İstanbul, Turkey
}

Corresponding Author: H. İbrahim ÇELIK, hcelik@gantep.edu.tr

\begin{abstract}
Today, many quality controls processes that are controlled by Image Processing (IP) technique has achieved state-of-the-art performance for all technology field. The image processing technique, which provides as accurate objective evaluation as the long and tiring traditional measurement methods, is nowadays widely used in textile industry. In this study, an alternative image processing technique has been proposed against the conventional wickability measurement method of nonwoven fabrics especially used in the hygiene products. An average liquid spread area ratio of airlaid nonwoven fabric was calculated with the developed algorithm and the accuracy of the proposed technology was demonstrated.
\end{abstract}

\section{ARTICLE HISTORY}

Received: 01.07.2019

Accepted: 17.02.2020

\section{KEYWORDS}

Absorption, Spreding Area, Airlaid-nonwoven, Image Processing

\section{INTRODUCTION}

In recent years, approximately $33 \%$ of all produced nonwovens worldwide is used for hygiene products (i.e. feminine care, adult diapers and baby diapers) [1]. Air-laid nonwovens are mainly composed of wood pulp and have a nature of high absorbing performance. It can be mixed with a definite proportion of super absorbent powders (SAP), super absorbent fibers (SAF), polyethylene powders or bicomponent fibers to improve its absorbing capabilities. Air-laid technology has some advantages compared to other nonwoven technologies. Both natural and synthetic fibers can be used for manufacturing of air-laid nonwovens. On the other hand, fluff pulp that is the main raw material of air-laid nonwoven products is cheaper than other mostly used nonwoven raw materials. These products are used in number of different products across a wide range of industry's including; baby diapers, sanitary napkins, cleaning cloths, wipes, cosmetic removal pad, geotextiles, covering materials, and upholstery [2-5].

Since average skin temperature and sweat loss are two physiological parameters that affect the heat balance of body metabolism, these parameters have great effect on the comfort of people. Accordingly, perceptions and evaluations of the comfort of clothes are influenced by environmental factors such as ambient temperature and humidity. Clothing which are next to skin should be capable of transferring moisture from the skin to the outer surface quickly and evaporating quickly to keep the body

To cite this article: Çelik HI, Gültekin E, Nohut S. 2020. Development of an algorithm to determine the liquid spreading area of airlaid nonwoven fabrics by image processing method. Tekstil ve Konfeksiyon, 30(2), 83-91. 
dry and cool. Depending on these effects, it is expected that the air-laid nonwoven fabrics exhibit quite higher absorbency due to their porous structure and raw material inherent characteristics. The critical comfort performance parameter of textile products is their liquid absorption and spreading capacity. Liquid absorption and spreading of water is influenced by fiber type, fiber fineness, fabric structure and mechanical or chemical finishing process applied on the fabric. The liquid transportation in fabric structure also depends on fiber crystalline structure, crystalline size, orientation and distribution. The amorphous region properties of the fiber and liquid properties such as viscosity and evaporation have also important effect on liquid transportation [6].

Many of the previous studies are generally related to examination about comfort and absorbency properties of fabric performance, depending on the porosity of the nonwoven fabric structure. The literature search put forward that the absorbency is one of the important properties that depends on the nonwoven fabric's porous structure, which is measured as vertical capillary (wicking), horizontal wicking and glass tube wicking. These test methods are applied to baby diapers and sanitary napkin, especially for hygiene purposes [6-11].

Absorbency or absorption capacity of a textile product can be evaluated by using different methods; liquid absorbency, liquid absorptive capacity and the liquid wicking rate (capillarity) (NWSP 010.1.RO (15) Edana Standard) [7] also absorption time rate AATCC 79-2007 standard [8]. All these test methods are based on falling a water drop on the fabric specimen or immersing the fabric specimen in a water tank. Thus, the water transmission and liquid management of the samples are assessed. When we examine the measurement principles of the standard methods, it can be seen that these measurements are made by visual assessment. There are some difficulties to determine wickability property of fabrics. The visual evaluation of the liquid rise on the fabric, the deficiency of measurement results for dark coloured fabrics, and especially the replacement of test sample for repeated measurement and time-consuming test procedure [14]. Several methods have been reported in literature in order to overcome these difficulties. Some studies have also concentrated on a test apparatus based on electromagnetic field induction, and the distance of liquid rise as a function of time has been measured using the apparatus [13-12]. Furthermore, for hygiene products or for other textile products that are expected high absorption capacity the liquid spreading area and spreading rate are also important parameters. For the measurement of spreading area and rate, different experimental approaches have been introduced in literature [12-15].

Apart from mentioned previous study, there are some studies about the wickability or absorbency measurements of fabrics using image processing techniques [16-22]. D.Brook and friends proposed that a wicking test method using an image analysis technique was develop to determine longitudinal strip wicking measurement automatically. Weft knitted fabrics used to determine wicking mechanism. The method based on the idea; the image acquired after the fabric had started wicking. They were focused on the partition the image into a set of regions along the fabric strip. It was stated that the accuracy of the measurement comparison between automatic and manual method obtained almost the same result [17]. Xiao-Yun et all. [18] studied on the water transport measurement through the fabrics using image processing technique. In this study, after 30 minutes the samples were captured by camera and then divided into two areas to show the height of the vertical wickability. D. Raja and their friends [21] represented a measurement technique to capture the inplane liquid spread in fabric by image processing principle. The water spreading area was captured with digital camera and the spreading behavior of fabrics produced from single, double yarns and various combinations of these yarns were calculated automatically. In another study, D.Raja et all. [19] compared the accuracy of three new developed test methods; manual method, commercial image analysis method using Photoshop and embedded image analysis. They described the water spreading behavior of fabrics such as rate of the absorbency and total absorbent capacity. In this study, 12 different knitted/woven fabrics were used in order to compare these test methods. $20 \mathrm{~mm}$ diameter solid circle was drawn as a manual method and the circle image was taken by a camera. Then, the resolution of the image taken was changed in Photoshop, so that the area of the drawn circle was calculated in pixels. And the accuracy of the applied method was stated to be high. The correlation among the methods were investigated.

As we can conclude from the previous studies, objective and accurate water spreading methods for textile fabrics can be developed by using image processing techniques. So, both vertical and horizontal liquid transfer detection of fabrics with image processing technique has become widespread. In this study, a machine vision system was built and an image-processing algorithm has been developed to objectively measure and assess the water spreading capacity of nonwoven fabrics. The developed system was tested on air-laid nonwoven fabric samples and the success ratio of the system was determined by comparing the results with manual evaluation.

\section{MATERIALS AND METHODS}

\subsection{Material}

Airlaid nonwoven fabrics produced from wood pulp as raw materials and bonded with latex (ACRYPOL EXP. 9) technique were used in 3 different areal weights; 50 GSM, 55 GSM and 60 GSM (Gram per Square Meters-GSM). 
The average thickness values are $0.52 \mathrm{~mm}, 0.52 \mathrm{~mm}$ and $0.58 \mathrm{~mm}$ respectively. The cellulosic pulp, which is disintegrated with the help of air in the form of cardboard is shredded between $1-3 \mathrm{~mm}$, is distributed homogeneously on 3 feeder heads and spread on conveyor belts. In the following stage, compaction (compression cylinder) is applied to distribute the surface evenly and provide the desired thickness. The latex is then applied to the first surface by spraying and the product enters first oven. After exiting the product is calendered to ensure smoothness of the surface and to adjust thickness. The product returns to the rear surface by wrapping it in a cylinder and latex is applied to that surface. Then, it is entering the 2nd oven, the product returns to the first surface by means of a cylinder and passes through a third oven for a short time and comes to the winding unit (Figure 1).

\subsection{Methods}

Generally, in determining the liquid absorption performance of Airlaid Nonwovens, some methods are used to find out the time required for liquid absorption and spreading through the fabric structure. These tests are carried out in the form of vertical and horizontal capillarity. In the capillarity measurement methods, the application and evaluation procedures are achieved manually. Both vertical and horizontal capillaries are determined with vision evaluation. Any automatic fabric capillarity performance measurement system was not encountered. We considered that developing a machine vision system and an image processing algorithm may be a proper approach for objective, accurate and precise liquid spreading area measurement. For this aim, a prototype experimental set-up was built for dropping artificial blood on the fabric surface and acquiring the image frames after a spreading time. The details of the image processing algorithm and experimental set-up are given below.

\subsection{Image Acquisition Prototype System}

The spreading speed in the nonwoven fabrics are as important as the spreading area. Therefore, in the determination of the absorbency studies measured by the image processing technique, the measurement is achieved as a plot area versus time. However, since the absorption and liquid transmission rates of the hygiene nonwovens that are used as material in this study are very high, the liquid spreading limit is reached in very short time duration. So, we only concentrated on the liquid transmission area measurement.

In this study, a prototype machine vision system was set-up to acquire image frames of the nonwoven fabric samples to calculated spreading area (Figure 2). The image acquisition system consists of microscope camera (1000X 2MP Digital Microscope Camera), tripod, lightening unit, cabin, burette, support stand and computer. To eliminate the disturbing effects of external light, all the equipment expect computer were placed inside a cabin. All image frames were taken under a constant light intensity. As in many applications, camera view size can be adjusted according to the desired pixel size. In accordance with the required horizontal field of view, the working distance between the inspected surface and camera lens is determined (Figure 3). This information is used to obtain the image frame which want. The field of view of the camera can be calculated with following Equation (1).

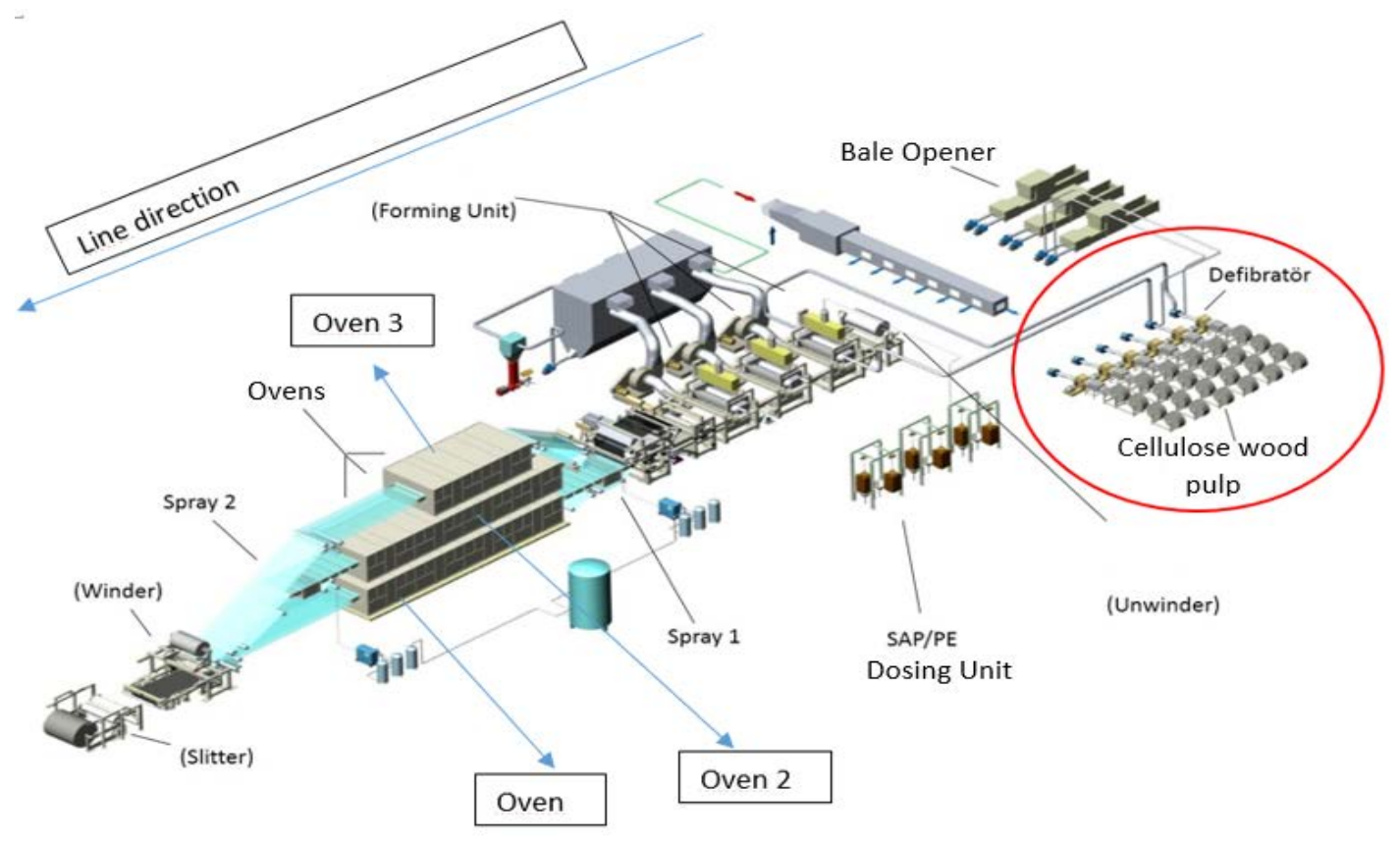

Figure 1. Airlaid Production Line 


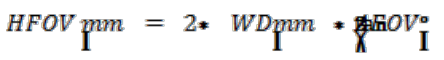

Herein, WD is the working distance from the lens, AFOV is the angular field of view and HFOV is the horizontal field of view [23].

According to HFOV equation, the distance between camera and fabric surface were adjusted via tripod so that a vision area approximately $2 \mathrm{~cm}$ x $1.5 \mathrm{~cm}$ was obtained. An adjustable syringe was used to ensure that constant amount of liquid was dropped. The microscope camera was connected to the computer and so the digital image frames size (480x640) were acquired and saved.

After 10 seconds, $0.2 \mathrm{ml}$ artificial blood was dropped on fabric sample, the image frame was acquired. This time duration was determined by testing on all samples and observing the maximum time needed for reaching maximum spreading area. For area measurement, 10 specimens with size of $10 \mathrm{~cm} \mathrm{x} 10 \mathrm{~cm}$ were prepared from different parts of each fabric sample. Totally 30 samples were obtained.

\subsection{Spreading Area Measurement Algorithm}

Image processing algorithm that determines the spreading area has been created in MATLAB environment. Images were first converted into gray level form. Later, to improve image visual quality, the noise in the image was cleaned by applying several image enhancement filters. By controlling the histogram, the contrast setting of the image was adjusted. After contrast setting step, the logarithmic transformation application was used, due to having the most effective role to find the spreading area. Transform intensity or gray-level transformation is called the simplest form of point-to-point transformation when the neighborhood of each point is of size 1 x 1 (a single pixel). The gray-level image has a very limited density range. To alleviate this problem and distribute densities over a wider range, the logarithmic and contrast stretching transformation can be applied as Equation (2). One of the principal's uses the log transformation is to compress dynamic range [24];

$$
g=c * \log (1+f)
$$

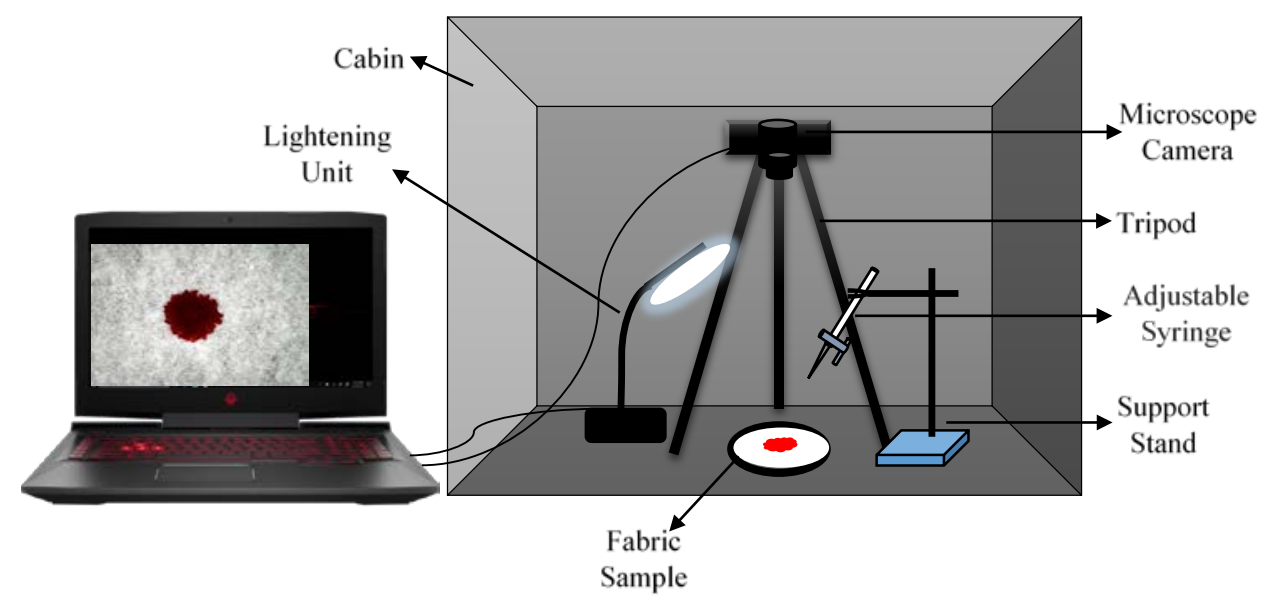

Figure 2. Schematic view of the system

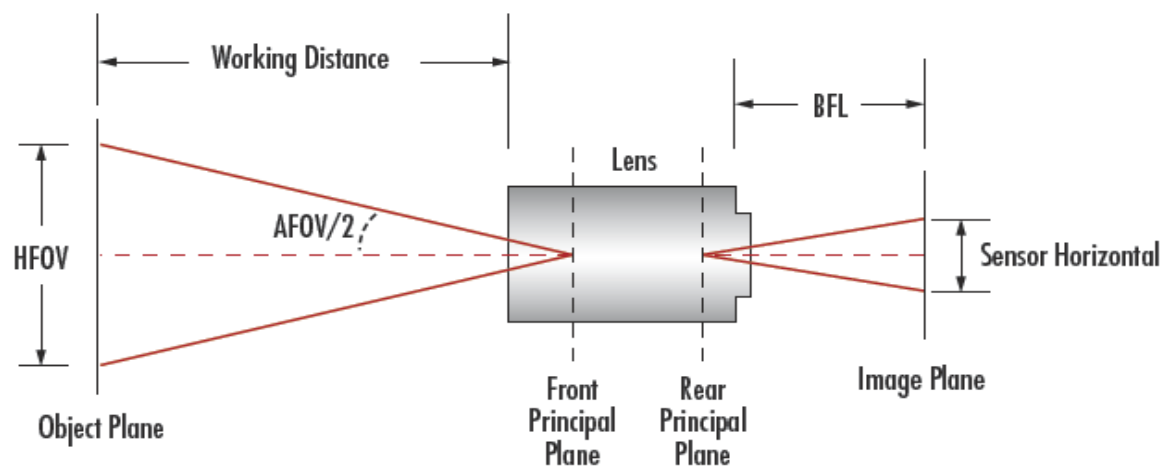

Figure 3. Relationship between HFOV, Sensor Size and WD for a given Angular FOV [23] 
where $\mathrm{c}$ is a constant and $\mathrm{f}$ is floating point.

In the transformation, firstly the image must be converted from unit8 to double image. Once the equation is applied to the full image, we can see a much larger area of the spectrum values in the results of logarithmic compression of the image new dynamic range. Since the large spectrum image has a very wide dynamic range, most of the spectrum is mapped to black tones. This conversion is used to expand dark pixels and compress the value of bright pixels. A value of 1 is added to each pixel values of the input image, because $\log (0)$ equals infinity if the image has a density of 0 pixels. The log contrast stretching part of the algorithm used in this study showed the most important effect. The effect of the Log transformation before the binarization process is given in Figure 4(b). As it can be recognized from the comparison between Figure 4(a) and Figure 4(b), the log transformation has significant effect on binarization and spreading area calculation accuracy.

After the finishing pre-processing stage, in order to obtain binary image, Otsu's thresholding was applied to the enhanced image. Otsu's thresholding method is used to distinguish the object from the background and insulate only object (foreground) from the image. The image frames are converted into the binary form of black (0) and white (1). According to this, Otsu's method divides the image into

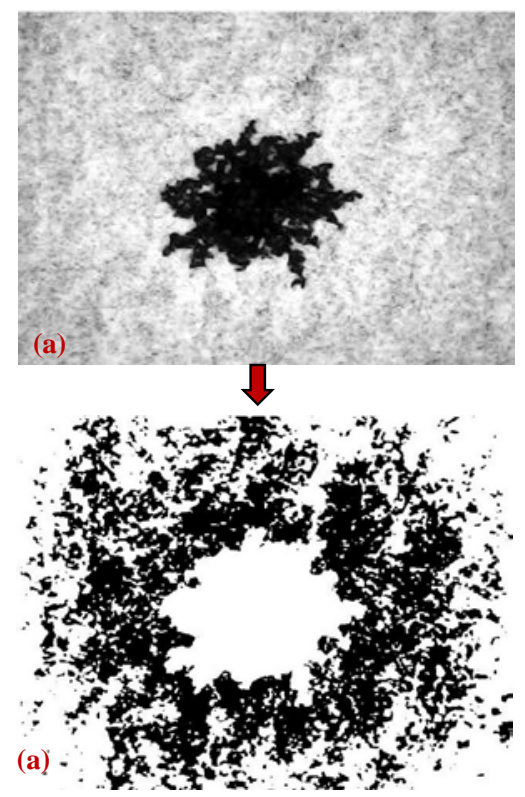

two parts, white and black $\left(\mathrm{T}_{0}\right.$ and $\left.\mathrm{T}_{1}\right)$. Here $\mathrm{T}_{0}$ is consist of intensity level from 0 to $t$ or in set notation $\mathrm{T}_{0}=\{0,1,2, \ldots, t\}$ and region $\mathrm{T}_{1}=\{t, t+1, \ldots, ., l-1, l\}$ where $t$ is the threshold value, $l$ is the image maximum gray level. In Otsu method, it is based on calculated variance statistics of $\mathrm{T}_{1}$ and $\mathrm{T}_{0}$ sets. The optimum threshold level of the image is determined by the calculated variance values [25].

For labeled boundary of the spreading area, mathematical morphology is used to extract image detail based on enhancement processes such as skeletonizing, thinning, thickening, and convex hull and object marking properties of form or shape within the image. The image preprocessing and post-processing such as noise filtering, shape simplification and object segmenting from the background are also able to be achieved by means of morphological operations. The main morphological operations consist of 'dilation', 'erosion', 'opening' and 'closing'. For this study, opening operation used to extract the area. Opening is a morphological operation of erosion followed by dilation with the same structuring element. Opening of A by the structuring element B is denoted as in following Equation (3). The opening operation removes small, isolated objects from the foreground of an image, place them in the background [25].

\section{$A \cdot B=(A \ominus B) \oplus B$}

(b)

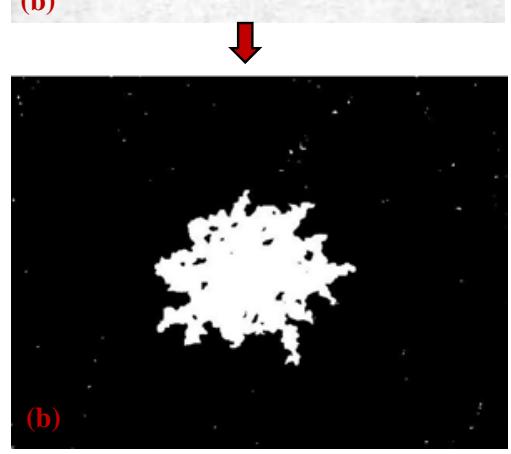

Figure 4. Transform intensity of the gray image (a) Log transform is not applied before binarization, (b) Log transform is applied before binarization

Above equation has a simple geometric interpretation: $A \circ B$ is the union of all translations of $\mathrm{B}$ that fit entirely within $\mathrm{A}$.

After all these operations, the area occupied by liquid was measured as a ratio of white pixels that corresponds to liquid transmission to total frame size as Equation (4).

Image Frame Area $=x * y$

$$
\text { Spreading Area Ratio }(\%)=\frac{\text { Swhite pixels in binary image }}{\text { Image Frame Area }} \times 100
$$

Where; $\mathrm{x}, \mathrm{y}$ are the sizes of the binary image. 
As a result, the flow chart of the algorithm is basically introduced in Figure 5. The execution time of the algorithm is approximately 15 seconds with $2.60 \mathrm{GHz}$ computer processer capacity.

Figure 6 shows the random application of spreading area measurement algorithm to each GSM sample image.

After all these processes, in order to assess the success rate of the proposed new algorithm, a comparison method that is based on the manual area calculation was also generated. In this manual area calculation method (Figure 7), first of all the the images taken by microscope camera were print out from the printer onto the millimetric papers, then the total sample area was measured with a ruler, and then each occupying the millimetric square in the spreading area was counted. Then, the number of black squares that displays the area of the absorption were counted. Finally, the spreading area of the liquid was determined as the percentage of black squares to total sample size (Figure 10). Thus, the accuracy of the new developed image processing algorithm was validated with manual area counting method.

\section{RESULTS AND DISCUSSIONS}

For the validation and success evaluation of the image processing algorithm, 10 tests were applied for each nonwoven fabric GSM. Totally, 30 absorption tests were performed. The results were determined with both algorithm and manual counting method. The comparisons of these two methods are given in Table 1 .
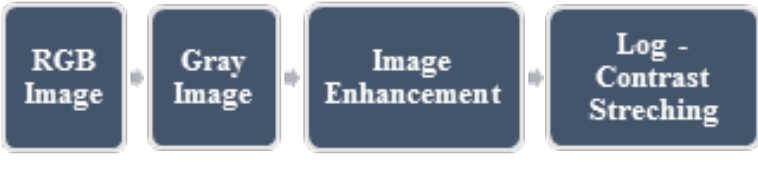

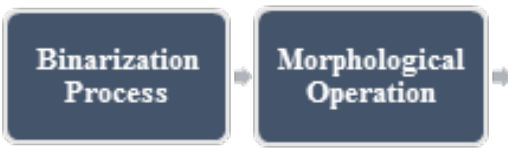

Area Detection and Percentage

Figure 5. The flow-chart of the algorithm
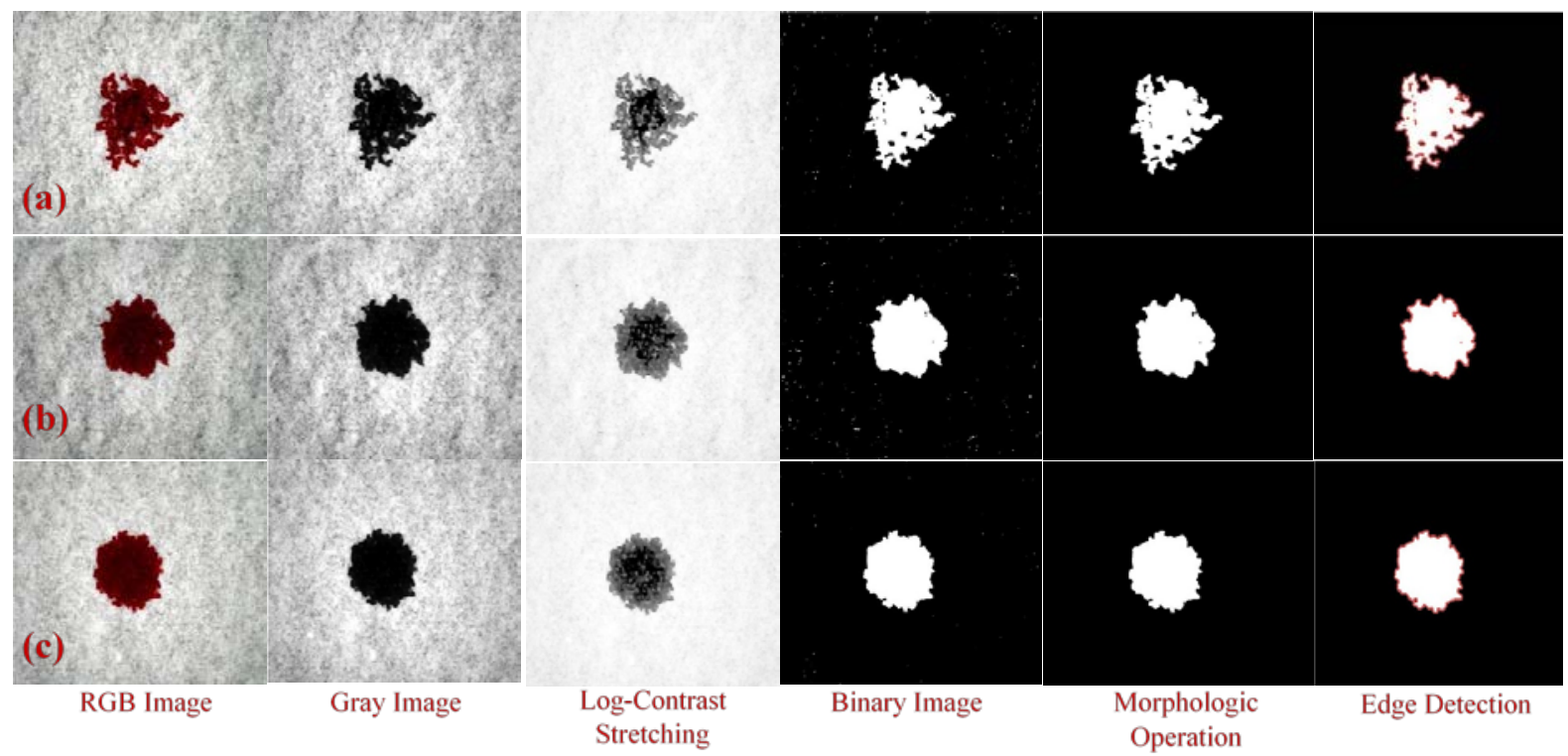

Figure 6. Results of the algorithm applied on the absorbed area (a) 50 GSM (b) 55 GSM (c) 60 GSM

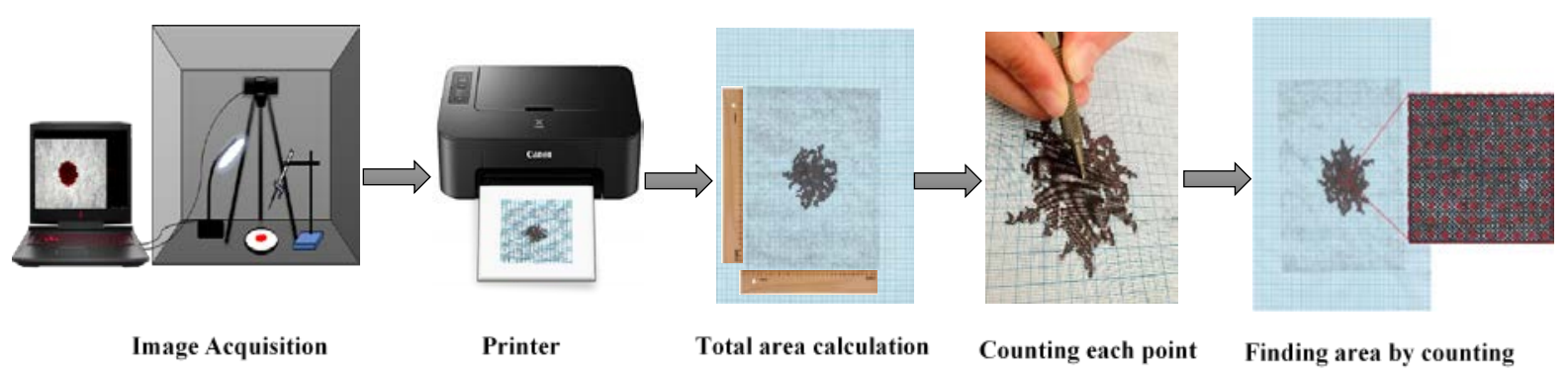

Figure 7. The process of checking the accuracy of the algorithm 
Table 1. Comparison of the spreading area calculation methods

\begin{tabular}{|c|c|c|c|c|}
\hline \multicolumn{2}{|c|}{ Fabric Samples } & \multirow{2}{*}{$\begin{array}{c}\begin{array}{c}\text { Spreading Area, } \\
\text { \% (IP Results) }\end{array} \\
6.90\end{array}$} & \multirow{2}{*}{$\begin{array}{c}\begin{array}{c}\text { Spreading Area, } \\
\text { \% (Manual Results) }\end{array} \\
6.75\end{array}$} & \multirow{2}{*}{$\begin{array}{c}\text { Err \% } \\
-2.290\end{array}$} \\
\hline 50 GSM & 1 & & & \\
\hline & 2 & 7.81 & 7.61 & -2.618 \\
\hline & 3 & 6.07 & 6.05 & -0.326 \\
\hline & 4 & 6.14 & 6.19 & 0.840 \\
\hline & 5 & 5.69 & 5.61 & -1.456 \\
\hline & 6 & 7.24 & 7.29 & 0.660 \\
\hline & 7 & 6.57 & 6.41 & -2.541 \\
\hline & 8 & 6.74 & 6.61 & -2.015 \\
\hline & 9 & 8.17 & 8.1 & -0.851 \\
\hline & 10 & 7.29 & 7.28 & -0.196 \\
\hline \multirow[t]{10}{*}{55 GSM } & 11 & 6.19 & 6.19 & 0.040 \\
\hline & 12 & 6.76 & 6.72 & -0.534 \\
\hline & 13 & 6.64 & 6.62 & -0.361 \\
\hline & 14 & 7.01 & 6.95 & -0.804 \\
\hline & 15 & 7.59 & 7.52 & -0.941 \\
\hline & 16 & 7.25 & 7.26 & 0.142 \\
\hline & 17 & 5.08 & 5.18 & 1.873 \\
\hline & 18 & 5.23 & 5.24 & 0.281 \\
\hline & 19 & 5.13 & 5.08 & -0.937 \\
\hline & 20 & 5.39 & 5.52 & 2.426 \\
\hline \multirow[t]{10}{*}{60 GSM } & 21 & 5.16 & 5.13 & -0.600 \\
\hline & 22 & 5.70 & 5.66 & -0.624 \\
\hline & 23 & 6.32 & 6.31 & -0.154 \\
\hline & 24 & 6.18 & 6.15 & -0.472 \\
\hline & 25 & 6.69 & 6.71 & 0.331 \\
\hline & 26 & 6.56 & 6.47 & -1.369 \\
\hline & 27 & 6.23 & 6.18 & -0.733 \\
\hline & 28 & 5.81 & 5.86 & 0.927 \\
\hline & 29 & 7.14 & 7.16 & 0.279 \\
\hline & 30 & 6.53 & 6.53 & -0.049 \\
\hline
\end{tabular}

A high regression rate of $R^{2}=0.99$ showed that the calculated spreading area by using image processing technique is nearly same with manually calculated area on the milimetric paper (Figure 8). In a similar study submitted by D. Raja, $R^{\mathbf{2}}=0.89$ was obtained between absorbency measurement by the commercial image analysis method (Adobe Photoshop ${ }^{\circledR}$ ) and manual transverse water spreading method. It was stated that the proposed method took less than one minute to measure spreading area in unit $\mathrm{mm}^{2}$ by image analysis technique [19]. It was also concluded that the conventional manual method for water spreading area measurement takes very long time (5-6 hours for each samples) by naked eye [14].
When the spreading characteristics of the samples are compared according to GSM values, it can be seen that more fiber is included in the unit area with higher GSM and the spreading geometry resembles to round and smoother boundary is observed. Contrary to 55 and 60 GSM, as seen from Figure 6.a, the absorption boundaries of 50 GSM have a curved structure and branched appearance since 50 GSM fabric has structure that is more porous in the structure.

The measured absorption area values via the developed machine vision system and calculated absorption area values via manual counting were compared in Figure 9. It can be clearly followed from Figure 9 that both results have very near values and so they have the same trends. 
According to these results, it can be concluded that the developed machine vision system and image processing algorithm can be surely used for absorption area calculation of airlaid nonwoven fabrics. Thus, the absorption area measurement test can be performed in a shorter time than conventional method. On the other hand, the results can be obtained in higher accuracy and sensitivity (Figure 10).

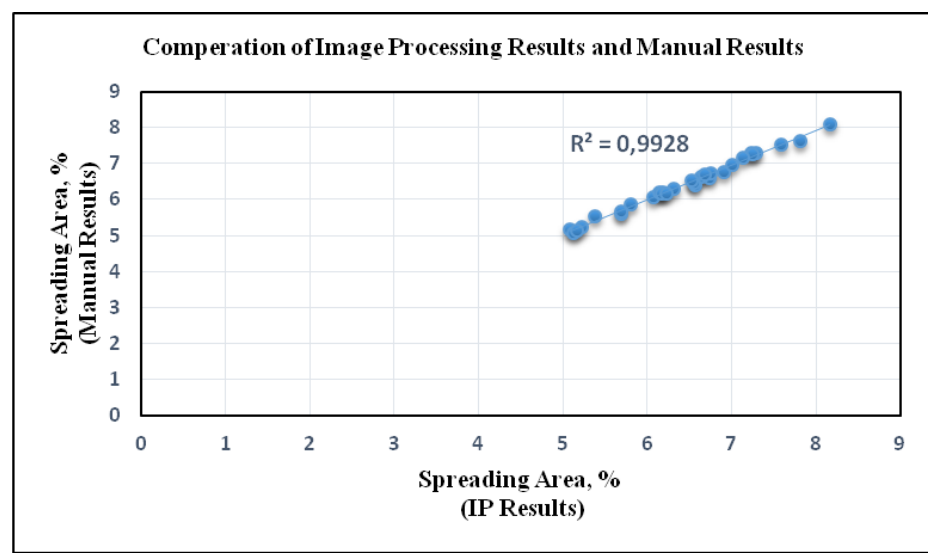

Figure 8. Regression graph of measurement methods

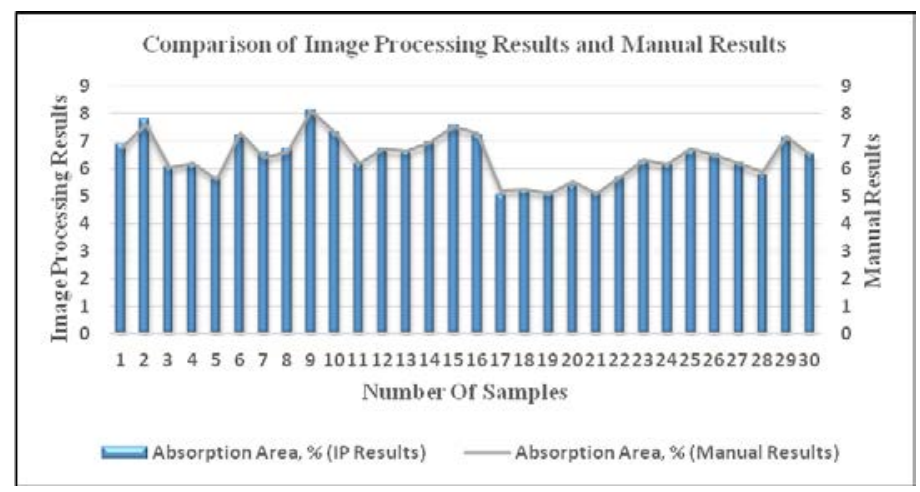

Figure 9. Comparison results of measurement methods
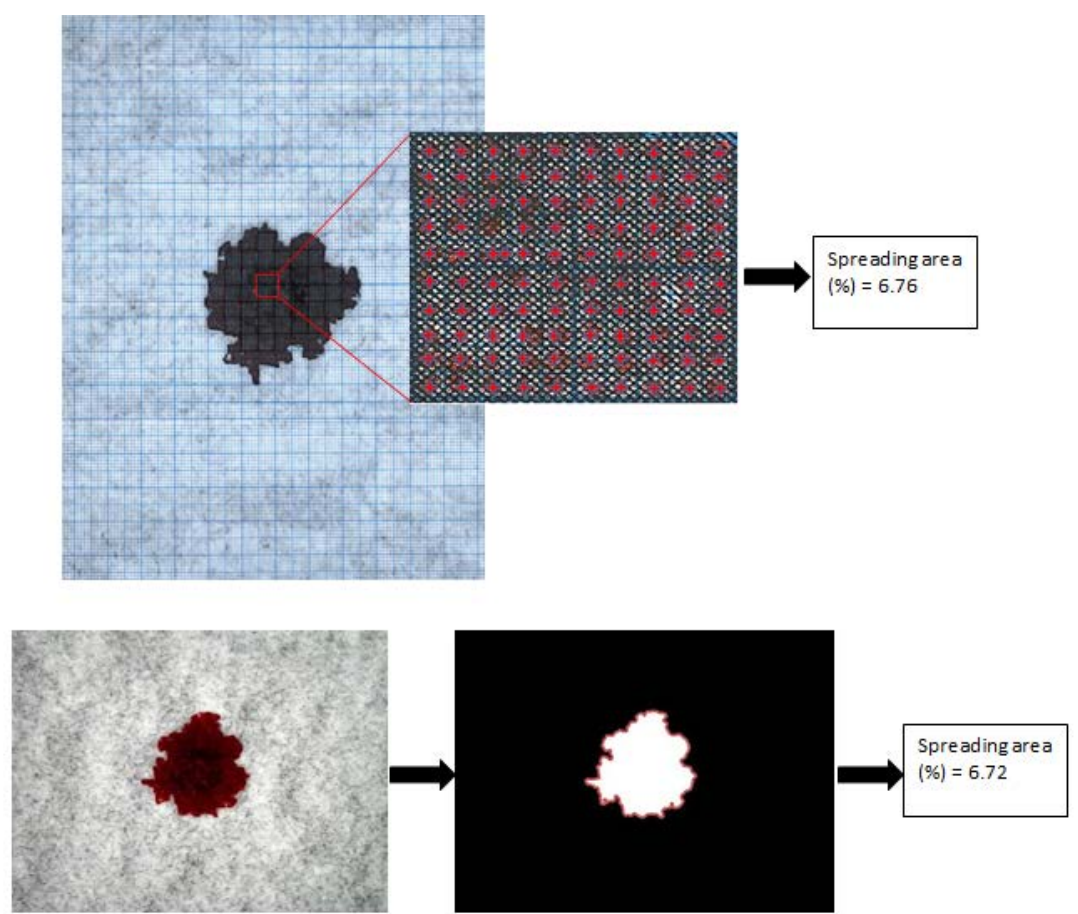

Figure 10. Comparison of liquid spreading behavior on the same fabric sample 


\section{CONCLUSION}

The random distributions of the fiber and the pore structure have an effect on the mechanical and comfort properties of nonwoven fabrics. Airlaid nonwoven fabrics are expected to have high absorbency properties. The wicking tests applied to these fabrics are time consuming during both the sample preparation process and the evaluation phase. Therefore, because of the widespread use of automation systems in the textile industry, a new method has been developed that can be adapted to the quality control process of an automatic measurement system. We have been able to express the percentage of area ratio given by image processing technique as millimeter square or centimeter square, which proves the accuracy of the proposed method.

\section{REFERENCES}

1. Sayeb S, Ben Hassen M, Sakli F. 2010. Study of some nonwoven parameters influence on the absorption kinetics of liquid. The Open Textile Journal, (3):1-5.

2. Çelikten E, Satıl EA. Nohut S, Elma KE. 2018. Development of Latex-Bonded Airlaid (LBAL) Nonwoven Fabric with High Wet Strength and Softness. Proceeding of $3^{\text {rd }}$ International Mediterranean Science and Engineering Congress (IMSEC 2018), (355) 967-969.

3. Irzmańska E, Dutkiewicz J. 2015. Preliminary evaluation of airlaid nonwovens with superabsorbent for use in protective footwear: tests involving a thermal foot model and climatic chamber. Fibres \& Textiles in Eastern Europe, 6(114):138-142.

4. Bartkowiak G. 2006. Liquid sorption by nonwovens containing superabsorbent fibers. Fibres and Textiles in Eastern Europe, 14(1):57.

5. Sadikoglu TG. 2005. Effect on comfort properties of using superabsorbent fibres in nonwoven interlinings. Fibres \& Textiles in Eastern Europe, 3(51):54-57.

6. PriyalathA S, Raja D. 2018. An Overview on Objective Evaluation of Wicking Property of the Textile Material Used in Sports. Trend in Textile Engineering \& Fashion Technology, 2(4)

7. EDANA Standard NWSP 010.1.RO (15)

8. AATCC Test Method 79-2007

9. Das A, Kothari VK, Makhija S, Avyaya K. 2008. Development of high-absorbent light-weight sanitary napkin. Journal of Applied Polymer Science, 107(3):1466-1470.

10. Rawal A, Kameswara RP, Russell V, Jeganathan S. 2010. A, Effect of fiber orientation on pore size characteristics of nonwoven structures. Journal of Applied Polymer Science, 118(5):2668-2673.

11. Aksoy A, Kaplan S. 2011. Tekstil materyallerinde sivi transfer mekanizmaları ve ölçüm yöntemleri, Tekstil Teknolojileri Elektronik Dergisi, 5(2):51-67.

12. Mazloumpour M, Rahmani F, Ansari N, Nosrati H, Rezaei AH. 2011. Study of wicking behavior of water on woven fabric using magnetic induction technique, The Journal of The Textile Institute, 102(7) 559-567.

13. Kumar B, Das A. 2014. Design and Development of a Computerized Wicking Tester for Longitudinal Wicking in Fibrous Assemblies. The Journal of The Textile Institute, 105(8):850-859
In this study, system design and algorithm of image processing technique, which is proposed as a new method in determination of liquid spreading property, is emphasized. The success of the method was taken into consideration rather than the technical properties of the material used. When compared to image processing technique, many quality control methods that are performed manually are very difficult and time-consuming processes. They also require attention and experience. For this reason, proposed image processing technique can be used to measure total absorbent capacity of the nonwoven fabric objectively and sensitively.

14. Atasağun HG, Okur A, Akkan T, Akkan LÖ. 2016. A test apparatus to measure vertical wicking of fabrics-a case study on shirting fabrics. The Journal of The Textile Institute, 107(12):1483-1489.

15. Birrfelder P, Dorrestijn M, Roth C, Rossi RM. 2013. Effect of fiber count and knit structure on intra-and inter-yarn transport of liquid water. Textile Research Journal, 83(14):1477-1488.

16. Patnaik A, Rengasamy RS, Kothari VK, Ghosh A. 2006. Wetting and wicking in fibrous materials. Textile Progress, 38(1):1-105.

17. Zhuang Q, Harlock SC, Brook DB. 2002. Longitudinal wicking of weft knitted fabrics: Part I: The development of an automatic wicking test method using image analysis. Journal of the Textile Institute, 93(1):88-96.

18. Jiang XY, Zhou XH, Weng M, Zheng JJ, Jiang YX. 2010. Image processing techniques and its application in water transportation through fabrics, Journal of Fiber Bioengineering and Informatics. 3(2):88-93.

19. Raja D, Ramakrishnan G, Babu VR, Senthilkumar M, Sampath MB. 2014. Comparison of different methods to measure the transverse wicking behaviour of fabrics. Journal of industrial textiles, 43(3):366-382.

20. Raja D, Koushik CV, Ramakrishnan G, Subramaniam V, Ramesh Babu V. 2012. Measuring in-plane liquid spread in fabric using an embedded image processing technique, Fibres \& Textiles in Eastern Europe, 20 4(93):72-76.

21. Memariani F, Ekhtiari E. 2010. Study on Wicking Measurement in Thin Layer Textiles by Processing Digital Images, International Journal of Engineering, 23(1):101-108.

22. Morent R, De Geyter N, Leys C, Vansteenkiste E, De Bock J, Philips. W. 2006. Measuring the wicking behavior of textiles by the combination of a horizontal wicking experiment and image processing, Review of scientific instruments, 77(9).

23. https://www.edmundoptics.com/resources/applicationnotes/imaging/understanding-focal-length-and-field-of-view/

24. Gonzalez RC. 2004. In Digital Image Processing Using MatlabGonzalez Woods \& Eddins, 84-86.

25. Yousefi J. 2011. Image Binarization using Otsu Thresholding Algorithm, University of Guelph, Ontario, Canada. DOI: 10.13140/RG.2.1.4758.9284 